FERMILAB-TM-2109

\title{
The Fermilab Computing Farms in 1999
}

\author{
Marina Albert et al. \\ Fermi National Accelerator Laboratory \\ P.O. Box 500, Batavia, Illinois 60510
}

May 2000 


\section{Disclaimer}

This report was prepared as an account of work sponsored by an agency of the United States Government. Neither the United States Government nor any agency thereof, nor any of their employees, makes any warranty, expressed or implied, or assumes any legal liability or responsibility for the accuracy, completeness, or usefulness of any information, apparatus, product, or process disclosed, or represents that its use would not infringe privately owned rights. Reference herein to any specific commercial product, process, or service by trade name, trademark, manufacturer, or otherwise, does not necessarily constitute or imply its endorsement, recommendation, or favoring by the United States Government or any agency thereof. The views and opinions of authors expressed herein do not necessarily state or reflect those of the United States Government or any agency thereof.

\section{Distribution}

Approved for public release; further dissemination unlimited.

\section{Copyright Notification}

This manuscript has been authored by Universities Research Association, Inc. under contract No. DE-AC02-76CH03000 with the U.S. Department of Energy. The United States Government and the publisher, by accepting the article for publication, acknowledges that the United States Government retains a nonexclusive, paid-up, irrevocable, worldwide license to publish or reproduce the published form of this manuscript, or allow others to do so, for United States Government Purposes. 


\title{
The Fermilab Computing Farms in 1999
}

\author{
Marina Albert, Troy Dawson, Jim Fromm, Lisa Giacchetti, Terry Jones \\ Tanya Levshina, Igor Mandrichenko, Ramon Pasetes, Marilyn Schweitzer \\ Karen Shepelak, Dane Skow, Stephen Wolbers
}

April 10, 2000

\section{Introduction}

The farms in 1999 changed in two major ways. First, PC's running Linux continued to expand and this allowed for the reduction of the SGI and IBM components of the farms. Second, the first large farms for CDF and D0 run II were purchased and installed in 1999. Simultaneously, a large increment for non-Run II computing was made.

The farms continue to provide large CPU resources for those experiments and calculations which benefit from this type of computing (large CPU, low I/O, dedicated resources). Farms usage will continue to increase given the demands of the user community (reflecting the scientific program) and the preparation for and beginning of Run II.

\section{The year in review}

The original PC farm (fnpc1-37) continued to provide large computing for the user community during 1999. These systems ran well and required little maintenance during the year. The I/O system for these machines (fnsfh) was augmented to provide adequate disk storage and tapedrives but otherwise was stable. The success in using the PC farm allowed us to decommission all of the IBM farms, including its I/O node and all of the single-processor SGI farms. The 4-processor O200 farm nodes were kept as part of the farm.

A major acquisition of farm computing was made in the summer/fall of 1999. 150 dual-Pentium III/500 MHz PC's were purchased after extensive pre-qualifying and an RFP. The resulting purchase consisted of 100 PC's from HiTec and 50 from Eternal Graphics. The PC's came with 1-6GB and 2-18GB diskdrives, $512 \mathrm{MB}$ of memory, and 10/100 Mbit ethernet interfaces. The default allocation is for 50 nodes 
to be dedicated to CDF, 50 to D0 and 50 to the rest of the laboratory's scientific program. In addition I/O nodes for the workers were acquired or upgraded. An SGI Origin 2000 was upgraded to serve as the I/O node for the 50 "general" nodes, an SGI Origin 2000 was acquired for the D0 I/O node and an SGI Origin 2200 was acquired as the CDF I/O node. Having a similar architecture for the I/O nodes is important to help reduce the support load and increase the ability to debug and solve problems in a common way across the farms. In addition, major switch acquisitions and installations were made during 1999 to give the worker and I/O nodes connections to the network with the required bandwidth.

The utilization of the farms during 1999 was lower than usual. The demand for the computing was not high enough during 1999 to use the full resource. Nevertheless, E871 finished their reconstruction of the 1996-97 data during 1999. This was a successful reconstruction and allows them to analyze the large sample of data that was acquired and get ready to reconstruct the data that was taken in 1999. E831 finished up some reprocessing and second-pass processing on the farms in 1999. No other major data reconstruction occurred in 1999 on the farms.

$\mathrm{NuTeV}$ ran simulation jobs during the last part of 1999 and made heavy use of the PC farms. A large sample of events was generated and this generation continues. Auger used some of the farms nodes to simulate high energy air showers. This computing is also run on FNALU. Most of the Auger work on the farms finished in mid-1999 but it may return if necessary. NUMI made small use of the farms for various calculations.

Two other large calculations were performed on the farms in 1999. The first was a calculation of beam dynamics in the Tevatron. This calculation took some time to optimize for the farms environment, due to some synchronization issues, but by the end of the year it was running smoothly on the 5 O200's left in the farm. There was a short but significant calculation performed by Walter Giele of the Theory Department during the middle of 1999.

\section{CPU utilization}

Table 1 provides the summary of CPU time (in VUP-equivalent units and in SpecInt95) for the whole farm in 1999. A plot of the CPU utilization (including all previous years of the UNIX farms) is shown in Figure 1.

Table 2 and Figure 2 show the utilization for each of the many experiments that have used the farms during 1999. E871 was the largest user of the farms. NuTeV and Auger simulations were also significant users of CPU on the farms. Theory 
calculations, E831 cleanup, and Tevatron calculations were the remaining large users of the farm in 1999.

Table 3 is a sum of all the CPU time used by all the experiments that have used significant CPU time on the farms during the last 9 years, along with the totals used by each. E831 is the largest farm user, with E871 second. Given the huge new capacity that has been added to the farms in 1999 (84,600 MIPS or 6180 SpecInt95) the list of integrated CPU will change dramatically once the new systems are fully integrated and utilized.

\section{SpecInt95 and MIPS}

The unit that we have been using to report utilization (the MIP) was and continues to be defined by us by running a simple program (called TINY). The program is an HEP track generator which subsequently performs pattern recognition and fits the tracks. This program has served us well, especially when the vendors' claims were exaggerated, highly tuned and otherwise not always trustworthy. The SPEC suite (see www.spec.org) improved and has been able to provide reasonably reliable and complete ratings for machines. We have decided to report 1999 in SpecInt95 units, as well as MIPS. SPEC has a new unit, Spec2000, which is just now being used. It is not clear yet how soon Spec2000 will become useful for the purposes of reporting CPU time. For now a switch to SpecInt95 will be made using published numbers. Table 4 gives the 1999 utilization and the integrated 1991-1999 utilization in SpecInt95 units. The factor used to convert from MIPS to SpecInt95 is 0.062653 . Please note that this factor is good only for the farms and its mix of CPU types. Every other system at Fermilab will have to use its own conversion factor to connect old (MIPS or VUPS) data with new (SpecInt95) data. 
Table 1 - Total CPU use on the Farms - 1999

$\underline{\text { Month }}$

CPU delivered

(Vax-Months/month)

(SpecInt95)

January

11315

February

$9247-579$

March

$5518-346$

April

$7987-500$

May

9084

569

June

6928

434

July

8453

530

August

6349

398

September

3750

235

October

4293

269

November

6088

381

December

4916

308

Table 2 - CPU use by experiment -1999

Experiment

$\underline{\mathrm{CPU} \text { time }}$

(Vax-years)

(SpecInt95)

E871

3333

209

$\mathrm{NuTeV}$

1280

80

Auger

834

52

Theory

568

36

E831

459

29

Beams

259

E872

32

16

NUMI

26

2

TOTAL

6791

425 
Table 3

Integrated Farm Use

(In units of MIP-years)

Through December, 1999

\begin{tabular}{|c|c|c|c|c|c|c|c|c|c|c|}
\hline Experiment & 1991 & 1992 & 1993 & 1994 & 1995 & 1996 & 1997 & 1998 & 1999 & Total \\
\hline E831 & & & & & & & 234 & 5988 & 459 & 6681 \\
\hline E871 & & & & & & & 191 & 1349 & 3333 & 4873 \\
\hline D0 offline & & 59 & 570 & 1072 & 1184 & 1743 & 96 & & & 4724 \\
\hline E706 & 28 & 82 & 732 & 992 & 795 & 686 & 19 & & & 3335 \\
\hline E791 & & 100 & 1232 & 1249 & 214 & 11 & & & & 2806 \\
\hline Auger & & & & & & & 880 & 1023 & 834 & 2737 \\
\hline $\mathrm{CDF}$ & & 110 & 320 & 438 & 752 & 956 & & & & 2576 \\
\hline E781 & & & & & & & 302 & 1566 & & 1868 \\
\hline $\mathrm{NuTeV}$ & & & & & & & & & 1280 & 1280 \\
\hline D0 MC & 101 & 162 & 396 & 197 & 108 & 23 & & & & 987 \\
\hline E665 & 14 & 105 & 733 & 91 & 2 & 10 & & & & 955 \\
\hline Theory & & & & & & & 309 & & 568 & 877 \\
\hline E771 & & 94 & 211 & 339 & 219 & & & & & 863 \\
\hline Beams & & & & & & & 105 & 112 & 259 & 476 \\
\hline E866 & & & & & & 55 & 409 & 8 & & 472 \\
\hline NUMI & & & & & & & & 412 & 26 & 438 \\
\hline E789 & & 156 & 247 & & & & & & & 403 \\
\hline E687 & 99 & 235 & 29 & 30 & 2 & & & & & 395 \\
\hline Minos & & & & & & & 221 & & & 221 \\
\hline Recycler & & & & & & 61 & 130 & 19 & & 210 \\
\hline E872 & & & & & & & 21 & 60 & 32 & 113 \\
\hline E760 & & 54 & & & & & & & & 54 \\
\hline E835 & & & & & & & & 52 & & 52 \\
\hline E731 & 38 & & & & & & & & & 38 \\
\hline Magnet & & & & & & & 28 & & & 28 \\
\hline Total & 267 & 1156 & 4541 & 4408 & 3276 & 3545 & 3217 & 10707 & 6791 & 38908 \\
\hline
\end{tabular}


Table 4

Integrated Farm Use

(In units of SpecInt95-years)

Through December, 1999

$\begin{array}{lrr}\text { Experiment } & 1999 & \text { Total } \\ \text { E831 } & 29 & 419 \\ \text { E871 } & 209 & 305 \\ \text { D0 offline } & & 296 \\ \text { E706 } & & 209 \\ \text { E791 } & 52 & 176 \\ \text { Auger } & & 171 \\ \text { CDF } & & 161 \\ \text { E781 } & 80 & 117 \\ \text { NuTeV } & & 80 \\ \text { D0 MC } & & 62 \\ \text { E665 } & 36 & 60 \\ \text { Theory } & & 55 \\ \text { E771 } & 16 & 54 \\ \text { Beams } & & 30 \\ \text { E866 } & 2 & 30 \\ \text { NUMI } & & 27 \\ \text { E789 } & & 25 \\ \text { E687 } & & 25 \\ \text { Minos } & & 14 \\ \text { Recycler } & & 13 \\ \text { E872 } & 2 & 7 \\ \text { E760 } & & 3 \\ \text { E835 } & & 3 \\ \text { E731 } & & 2438 \\ \text { Magnet } & & 2 \\ \text { Total } & 425 & \end{array}$




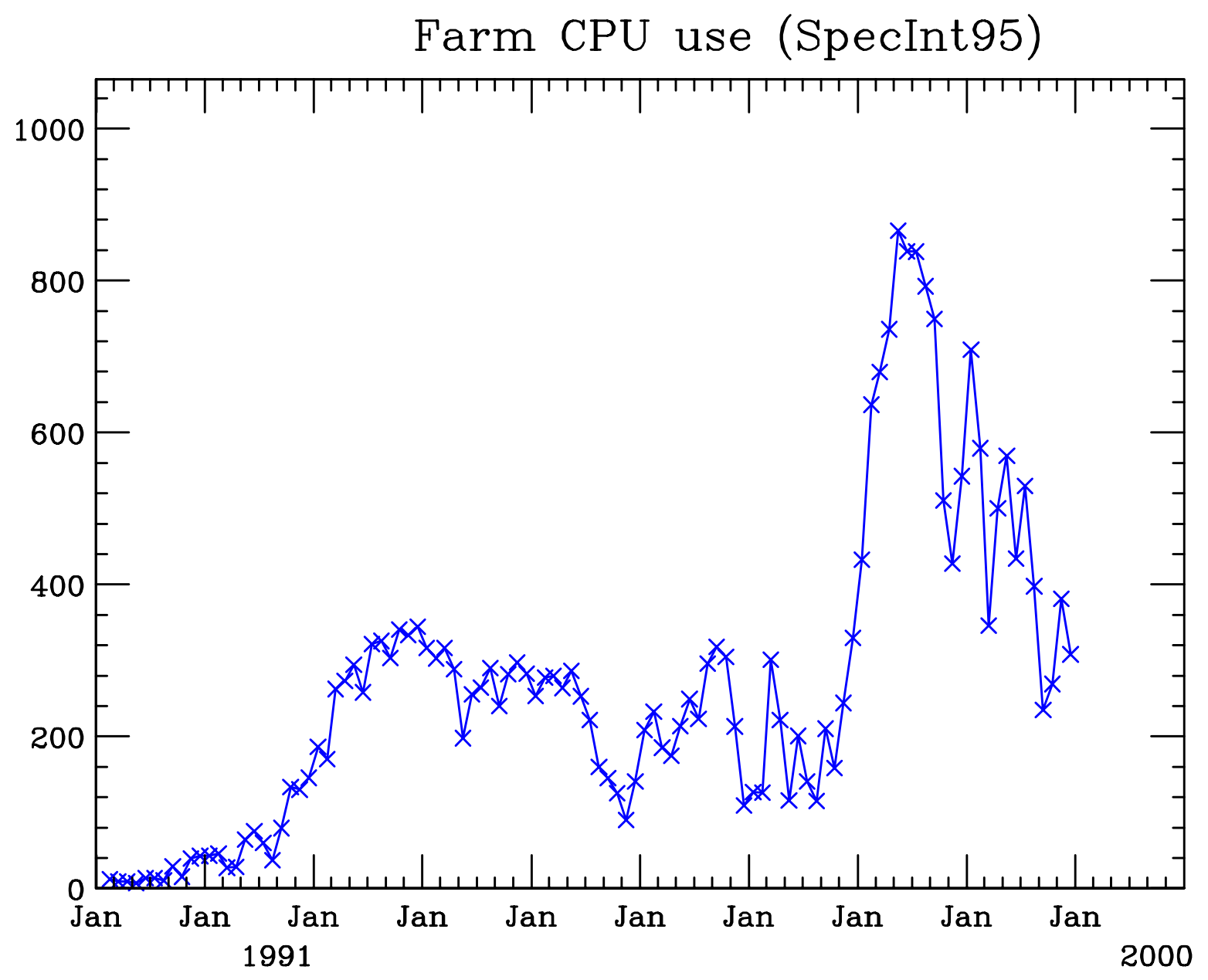

Figure 1. 


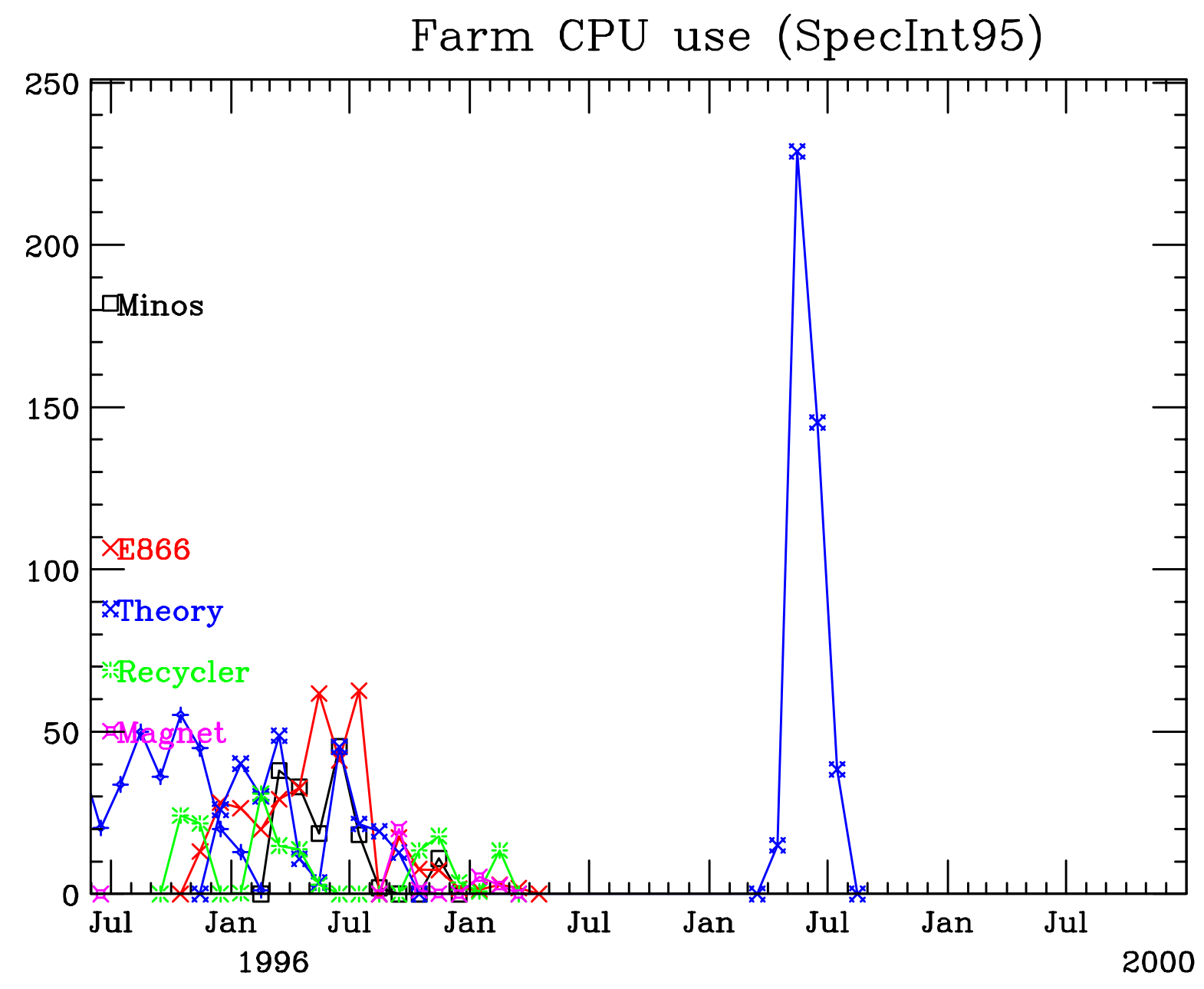

Figure 2(a). 


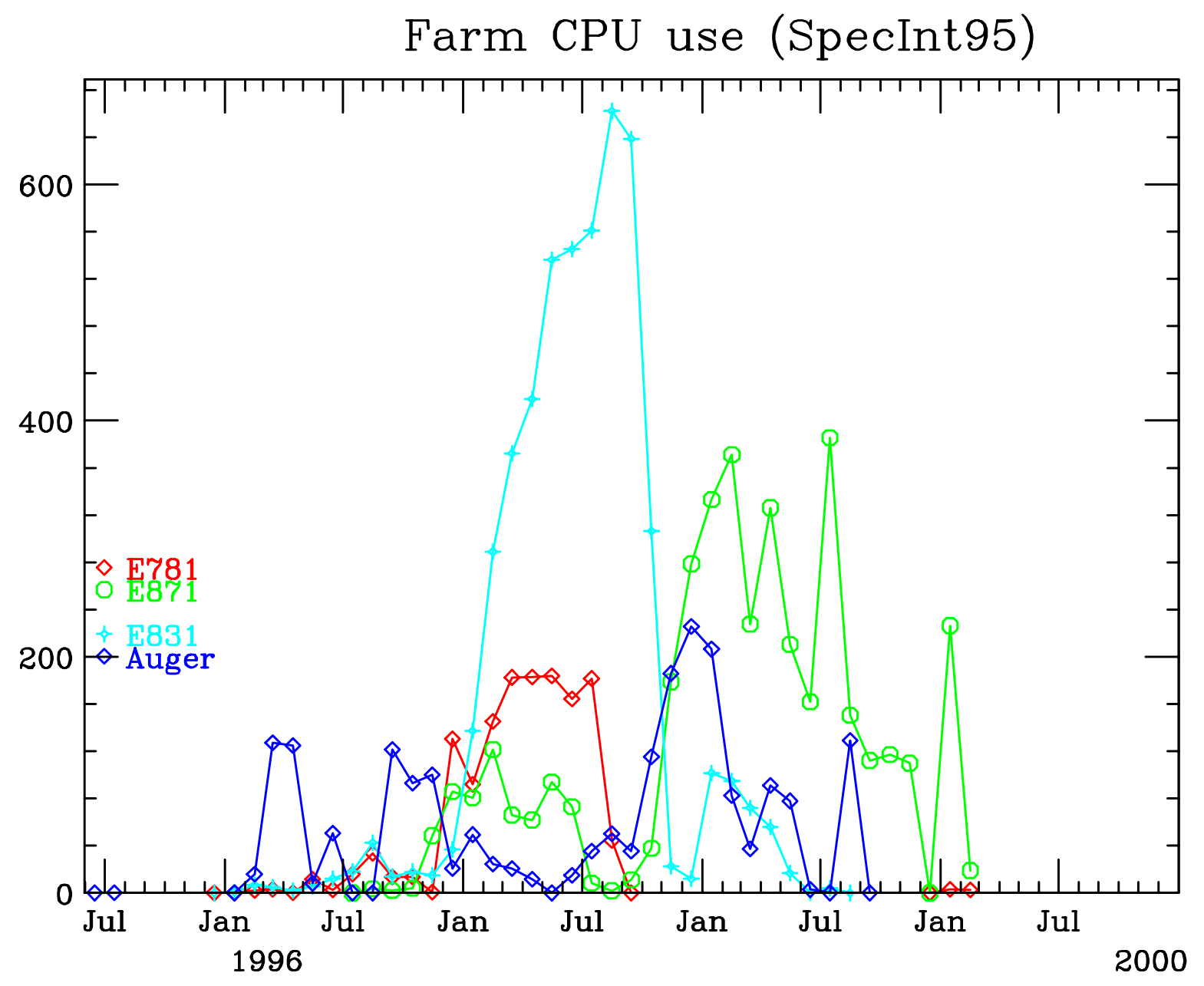

Figure 2(b). 


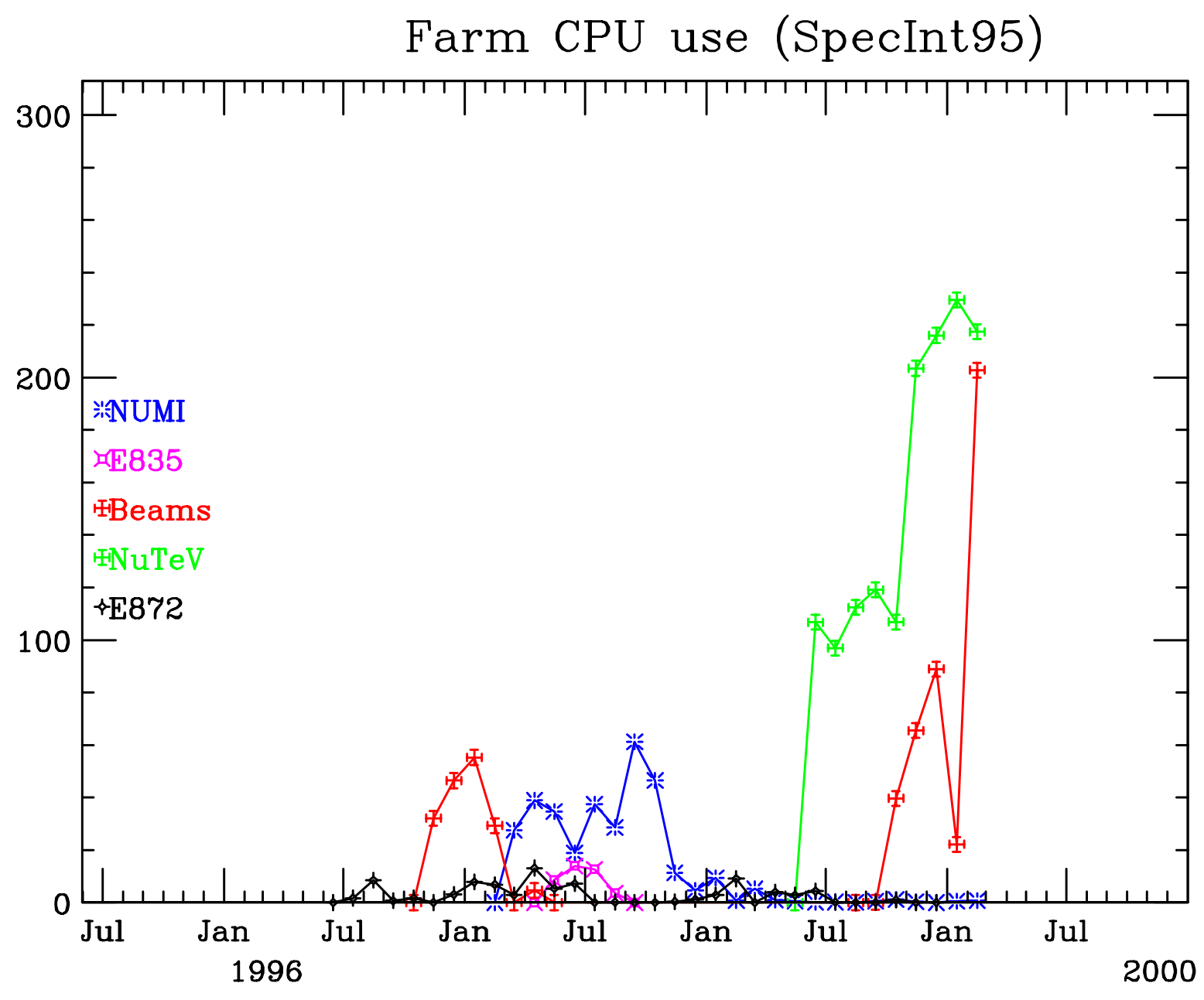

Figure 2(c). 


\section{Allocations}

For completeness the allocations of worker nodes as of March 24, 2000 is given in tables 5-6. These allocations are based on the laboratory's priorities, the ability of the group to use the resource and of course the need of the various groups for computing. These allocations are shifted fairly often to reflect the changes in the circumstances of the groups as they (hopefully) finish what they need to do and move on to other tasks.

Table 5

Current Farm Allocations

\section{Experiment}

CDF

D0

$\mathrm{BTeV}$

$\mathrm{KTeV}$

E871

Beams

$\mathrm{NuTeV}$

E781

NUMI

Linear Collider

TOTAL allocated

\section{Allocation in MIPS}

28200

28200

15228

8460

6696

4160

4092

3384

2232

1128

101780
Allocation in SpecInt95

1767

1767

954

530

420

261

256

212

140

71

6378 
Table 6

Current Farm Allocations

All Nodes

\begin{tabular}{|c|c|c|c|c|c|}
\hline Expt & O200 & $\mathrm{PC}(333)$ & $\mathrm{PC}(500)$ & MIPS & SpecInt95 \\
\hline $\mathrm{CDF}$ & & & 100 & 28200 & 1767 \\
\hline D0 & & & 100 & 28200 & 1767 \\
\hline $\mathrm{BTeV}$ & & & 54 & 15228 & 954 \\
\hline $\mathrm{KTeV}$ & & & 30 & 8460 & 530 \\
\hline E871 & & 36 & & 6696 & 420 \\
\hline Beams & 5 & & & 4160 & 261 \\
\hline $\mathrm{NuTeV}$ & & 22 & & 4092 & 256 \\
\hline E781 & & & 12 & 3384 & 212 \\
\hline NUMI & & 12 & & 2232 & 140 \\
\hline Linear Collider & & & 4 & 1128 & 71 \\
\hline unused or test & & 2 & & 372 & 23 \\
\hline TOTALS & 5 & 72 & 300 & 102152 & 6400 \\
\hline
\end{tabular}




\section{Plans}

2000 will see the ramp up of the use of the 150 new PIII/500 PC's which were purchased in 1999. 50 of them are connected to the general purpose or "fixedtarget farms", 50 of them are dedicated to CDF and 50 to D0. The 50 new and 37 original general purpose farms will be rapidly allocated to a combination of E871, E781, NuTeV, BTeV, KTeV, Beams and Linear collider calculations. The 5 O200's may be moved to FNALU to provide CPU in that environment or they may be decommissioned.

The CDF and D0 farms will be dedicated most of the time to those experiments for testing of rates, functionality, mock data challenges, generation of simulation samples, etc. Additional nodes will be purchased in late 2000 or very early 2001 for the beginning of the run, scheduled for March 1, 2001.

Additional nodes for general purpose users may be acquired during 2000, though this depends on the utilization and demand from the various scientific users at the lab. There are potentially very large demands from BTeV, Linear Collider, Muon Collider and vlhe calculations. 